\title{
Uso da Peritoneostomia na Sepse Abdominal
}

\author{
Laparostomy in Abdominal Sepsis
}

\section{JUVENAL DA ROCHATORRES NETO ${ }^{1}$, ADONAI PINHEIRO BARRETO ${ }^{2}$, ANACAROLINALISBOAPRUDENTE $^{3}$, ALLISSON MÁRIO DOS SANTOS ${ }^{4}$, RODRIGO ROCHA SANTIAGO $^{5}$}

\author{
${ }^{1}$ Chefe do Serviço de Coloproctologia da Universidade Federal de Sergipe, Professor Doutor e \\ coordenador do internato de clínica cirúrgica; ${ }^{2}$ Acadêmico do serviço de coloproctologia da Universidade \\ Federal de Sergipe; ${ }^{3}$ Residente do serviço de coloproctologia da Universidade Federal de Sergipe; ${ }^{4}$ Acadêmico do \\ serviço de coloproctologia da Universidade Federal de Sergipe; ${ }^{5}$ Médico do serviço de coloproctologia da \\ Universidade Federal de Sergipe.
}

NETO J R T, BARRETO A P, PRUDENTE A C L, SANTOS A M, SANTIAGO R R. Uso da peritoneostomia na sepse abdominal. Rev bras Coloproct, 2007;27(3): 278-283.

RESUMO: Dentre as modalidades terapêuticas da sepse abdominal, a peritoneostomia tem papel decisivo permitindo explorações e lavagens da cavidade de forma facilitada. Observamos pacientes com diagnóstico clínico de sepse abdominal internados no Serviço de Coloproctologia do Hospital Universitário da Universidade Federal de Sergipe, e que foram submetidos a peritoneostomia de janeiro de 2004 a janeiro de 2006. Foram avaliados quanto ao diagnóstico primário e secundário, tipo de peritonite secundária, antibioticoterapia, esquema de lavagens, tempo de peritoneostomia, complicações e desfecho. Estudamos 12 pacientes, com idade de 15 a 57, média de 39,3 anos. Diagnóstico primário: abdome agudo inflamatório em 6(50\%), abdome agudo obstrutivo em $\mathbf{2}(16,7 \%)$, abdome agudo perfurativo em $2(16,7 \%)$, fístula enterocutânea em $1(8,3 \%)$ e abscesso intra-cavitário em $1(8,3 \%)$. Diagnóstico secundário: perfuração de cólon em 4(33,3\%), abscessos intra-cavitários em 3(25\%), deiscências de anastomoses em $3(25 \%), 1(8,3 \%)$ com tumor perfurado de sigmóide e $1(8,3 \%)$ com necrose de cólon abaixado. Peritonite fecal em 10(83,3\%) e purulenta em 2(16,7\%). A antibioticoterapia teve duração média de 19 dias. Lavagens de demanda em 6(50\%), programadas em $\mathbf{4}(33,3 \%)$ e regime misto em $2(16,7 \%)$. O tempo médio de peritoneostomia foi de 10,9 dias (1-36). Como complicações: evisceração em $2(16,7 \%)$ e fistulização em $1(8,3 \%)$. Quatro pacientes evoluíram com óbito.

Descritores: Peritoneostomia, Bolsa de Bogotá, tela de polipropileno, peritonite, sepse abdominal.

\section{INTRODUÇÃO}

O tratamento da sepse abdominal é prioritariamente cirúrgico, agindo no controle da fonte de infecção, remoção e drenagem de seus produtos, aliado a antibioticoterapia, suporte ventilatório e hemodinâmico adequados ${ }^{(1)}$. Em alguns pacientes pode ser necessária, como abordagem cirúrgica, a realização da peritoneostomia para tratamento da infecção intra-abdominal, minimizando a intensidade ou reduzindo a ocorrência de síndrome da resposta inflamatória sistêmica (SRIS) $(2,3,4,5,6)$.

A realização da peritoneostomia tem sido preconizada desde 1979, seus defensores advogam que a técnica permitiria a ampla drenagem de secreção purulenta através da abertura da parede e também facilitaria a lavagem da cavidade peritoneal através de reoperações programadas ou de demanda, conforme a necessidade.

Neste procedimento os planos da parede abdominal não são completamente aproximados, permitindo uma regular inspeção do estado das alças e drenagem de conteúdo intra-cavitário, reduzindo a necessidade de transferência do paciente para o centro cirúrgico, minimizando os riscos cumulativos dos múltiplos transportes e anestesias ${ }^{(3)}$. Além do seu uso no tratamento da sepse abdominal, outras indicações desse procedimento são: na síndrome compartimental ab-

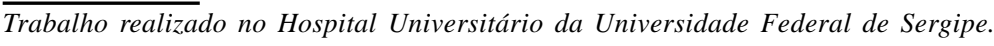

Recebido em 08/05/2007

Aceito para publicação em 04/06/2007 
dominal (1,3), "damage control" e fasciite necrotizante ${ }^{(1,4)}$. Tem, ainda, alta eficiência no controle da infecção abdominal e outras complicações da pancreatite necrotizante $^{(7,8)}$.

Analisaremos a utilização da peritoneostomia para controle da sepse abdominal, sendo utilizada a Bolsa de Bogotá associada ou não à tela de polipropileno com lavagens programadas a cada 48 horas, ou de demanda conforme indicasse o quadro clínico do paciente.

\section{PACIENTES E MÉTODOS}

O estudo foi realizado no Hospital Universitário da Universidade Federal de Sergipe de janeiro de 2004 a janeiro de 2006. Consistiu em uma análise descritiva retrospectiva de 12 pacientes que foram submetidos à técnica de peritoneostomia com Bolsa de Bogotá associada ou não à tela de polipropileno como estratégia terapêutica da sepse abdominal instalada nesses pacientes.

Os pacientes foram operados e acompanhados pela equipe de cirurgia coloproctológica desse Hospital. Eles foram analisados quanto ao diagnóstico primário, diagnóstico secundário, tipo de peritonite secundária, antibioticoterapia, esquema de lavagens, tempo de peritoneostomia, complicações e desfecho.

Os dados colhidos foram armazenados em planilhas do software Microsoft Excel 2003, com confecção de gráficos e tabelas.

\section{RESULTADOS}

Foram estudados 12 pacientes, $10(83,3 \%)$ homens e $2(16,7 \%)$ mulheres, com média de idade de 39,3 variando de 15 a 57 anos.

Com relação ao diagnóstico que constava como motivo da internação desses pacientes e suas intervenções cirúrgicas no nosso serviço, dos 12 pacientes estudados, 6 (50\%) tiveram como diagnóstico primário abdome agudo inflamatório, 2 (16,7\%) abdome agudo obstrutivo, 2 (16,7\%) abdome agudo perfurativo, 1 $(8,3 \%)$ fístula enterocutânea e $1(8,3 \%)$ paciente com abscesso intra-cavitário (Figl).

Após a impressão diagnóstica inicial, procuramos melhor estabelecer a razão da utilização da peritoneostomia. O diagnóstico firmado com a laparotomia nesses pacientes, ou seja, o diagnóstico secundário, foram perfuração de cólon em 4 (33,3\%) pacientes, abscessos intra-cavitários em 3 (25\%) pacientes, deiscências de anastomoses em 3 (25\%), um $(8,3 \%)$ paciente com tumor perfurado de sigmóide e um $(8,3 \%)$ outro com necrose de cólon abaixado ( $\mathrm{Ta}$ bela 1). Com relação ao tipo de peritonite secundária encontrada, a grande maioria, $10(83,3 \%)$ pacientes constituía-se de peritonite fecal, sendo que $2(16,7 \%)$ pacientes apresentaram peritonite purulenta.

A antibioticoterapia teve duração média de 19 dias, sendo utilizados sempre esquemas de amplo espectro visando Gram-positivos, Gram-negativos e anaeróbios. Quatro (33,3\%) pacientes foram submetidos ao esquema cefepima e metronidazol, 1 (8,3\%) paciente utilizou a associação cefepima, metronidazol e vancomicina, $1(8,3 \%)$ ciprofloxacina e metronidazol, $1(8,3 \%)$ cefepima, clindamicina e vancomicina, $1(8,3 \%)$ ceftriaxona e clindamicina, $1(8,3 \%)$ ceftriaxona e metronidazol e $1(8,3 \%)$ paciente utilizou cefepima associado ao metronidazol (Fig. 2).

Houve uma preferência na realização de lavagens da cavidade abdominal baseadas no quadro clíni-

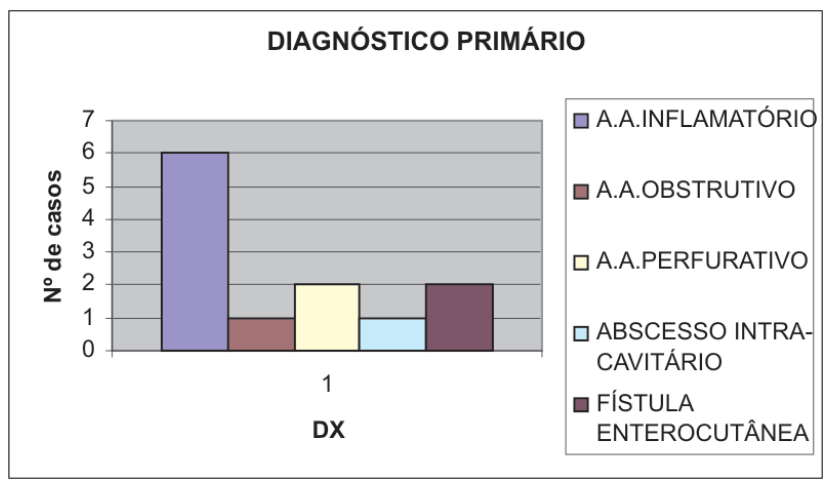

Figura 1 - Gráfico ilustrando a freqüência dos diversos diagnósticos primários.

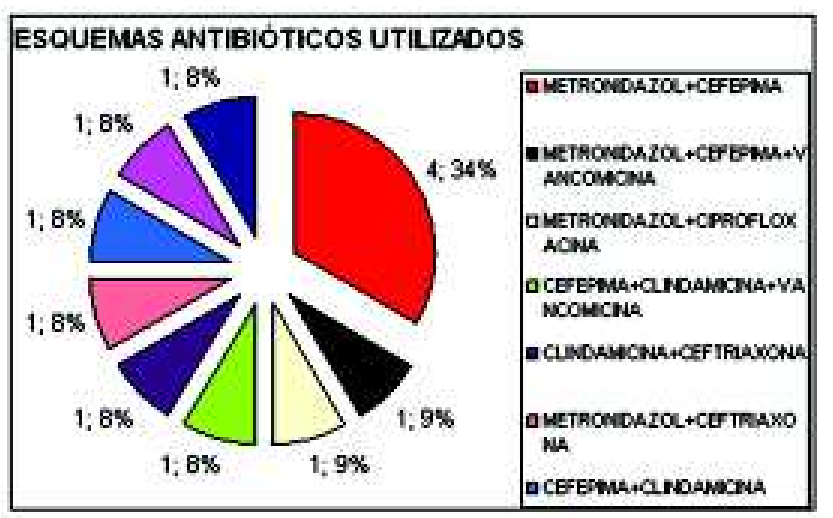

Figura 2 - Gráfico ilustrando os principais esquemas antibióticos utilizados. 
Tabela 1 - Tabela relacionando os diagnósticos secundários, bem como a freqüência de aparecimento deles.

\section{DIAGNÓSTICO SECUNDÁRIO}

TOTAL

\begin{tabular}{lr}
\hline A.A.PERFURATIVO & 4 \\
ABSCESSOS INTRA-CAVITÁRIOS & 3 \\
DEISCÊNCIA DE ANASTOMOSE (PÓS-COLECTOMIA TOTAL) & 1 \\
DEISCÊNCIA DE ANASTOMOSE (PÓS-DUHAMELL) & 1 \\
DEISCÊNCIA TOTAL DE BOLSA ILEAL & 1 \\
TUMOR PERFURATIVO DE SIGMÓIDE & 1 \\
NECROSE DE CÓLON ABAIXADO & 1
\end{tabular}

co-laboratorial do paciente, ou seja, sob regime de demanda em $6(50 \%)$ pacientes e a utilização do regime de lavagens programadas a cada 48 horas em $4(33,3 \%)$ pacientes. Houve ainda $2(16,7 \%)$ pacientes em que se optou inicialmente pelo esquema programado a cada 48 horas, mas que progrediram para lavagens de demanda. O tempo médio de peritoneostomia foi de 10,9 dias variando de 1 a 36 dias.

Como complicações do procedimento houve evisceração em 2 (16,7\%) pacientes e em 1 (8,3\%) paciente ocorreu fistulização. Quatro $(33,3 \%)$ pacientes foram a óbito por falência de múltiplos órgãos em decorrência do quadro séptico.

\section{DISCUSSÃO}

Em nosso estudo a peritoneostomia foi realizada como estratégia de controle da sepse abdominal em pacientes com complicações pós-operatórias colorretais, exceto um caso em que o foco da peritonite foi um tumor perfurado de sigmóide.

Diversos materiais e técnicas são descritos na literatura para a confecção da peritoneostomia, porém ainda não foi publicado um estudo prospectivo comparando-as, e a evidência de superioridade de qualquer uma delas ainda é baixa ${ }^{(4,8)}$. Utiliza-se geralmente uma tela (absorvível ou não) e/ou uma folha de material impermeável, esta com a vantagem de diminuir a perda hídrica e calórica ${ }^{(3)}$. As telas inabsorvíveis são constituídas à base de nylon, polipropileno ou Dacron, sendo associadas a altas taxas de fistulização que vão de 4 a $75 \%{ }^{(8)}$. As telas constituídas de materiais absorvíveis como poliglactin ou ácido poliglicólico parecem reduzir as taxas de surgimento de fístula ${ }^{(9,10,11,12)}$. Pode-se utilizar também o politetrafluoretileno (ePTFE), que é de consistência mole, flexível e desencadeia resposta inflamatória e reação de corpo estranho mínimas, tem também a grande vantagem de não aderir à superfície das alças diminuindo muito a ocorrência de fistulas ${ }^{\left({ }^{8}\right)}$. São descritas também técnicas que empregam o vácuo com tela de poliglactin e folha de material adesivo recobrindo a tela ${ }^{(3,10)}$ e técnica de bolsa-sanduíche com drenagem a vácuo que associa Bolsa de Bogotá e folha de poliuretano com taxas de morbimortalidades aceitáveis ${ }^{(13,14)}$. O uso da técnica com zíper associado à tela de polipropileno permite o fácil acesso à cavidade abdominal, otimizando as inspeções seriadas ${ }^{(15,16)}$. Utilizamos em nossa experiência a Bolsa de Bogotá para a confecção da peritoneostomia, que consiste na colocação de uma bolsa coletora de urina estéril, que tem baixo custo em relação a outros materiais descritos, e é um eficiente protetor contra as perdas de água e calor (fig. 3-4). Nós passamos a utilizar rotineiramente, como forma de reforço e contenção, a tela de polipropileno, que foi suturada à pele, juntamente com o bordo da bolsa coletora (fig.5). Passamos a fazê-lo em virtude de um paciente ter apresentado evisceração. São descritos na literatura que a evisceração e dificuldade de mobilização do paciente, constituem-se importantes problemas da utilização da Bolsa de Bogotá ${ }^{(3,11,12)}$.

A peritoneostomia leva a uma perda de fluidos, eletrólitos e calor, além disso, o risco de evisceração e isquemia intestinal é alto. Fístulas espontâneas desenvolvem-se em $16 \%$ dos pacientes, a ocorrência obrigatória de hérnia ventral e a demora na cura da ferida são observados ${ }^{(18)}$. Num estudo com 181 pacientes ocorreram fistulas gastrintestinais em 14\%, síndrome compartimental abdominal em 13\% e 9\% apresentaram deiscência ou evisceração ${ }^{(18)}$.

É relatada na literatura a ocorrência de fístula intestinal usando tela de polipropileno em $15 \%$ dos pa- 


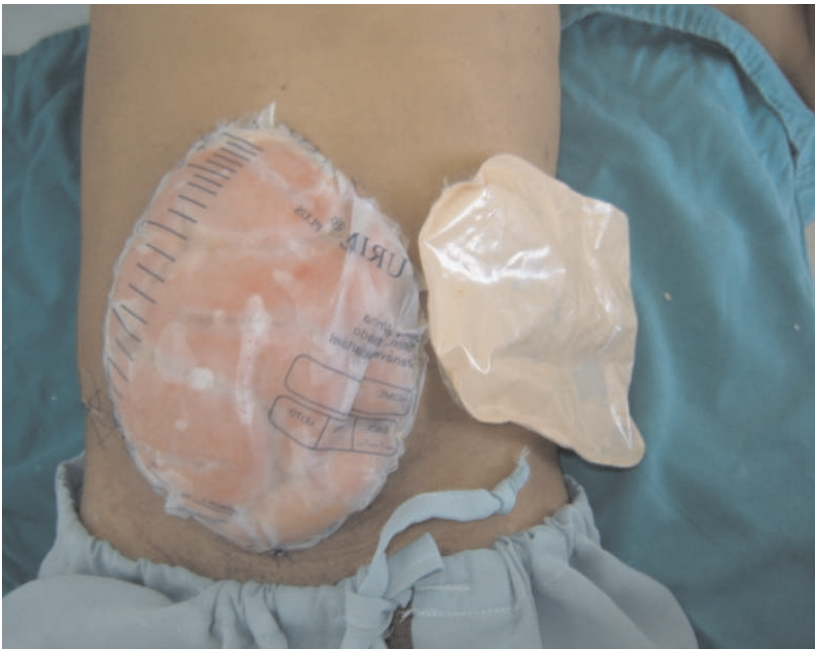

Figura 3 - Fotografia demonstrando paciente em $8^{\circ}$ dia de peritoneostomia com Bolsa de Bogotá, apresentando também ileostomia (visão superior).

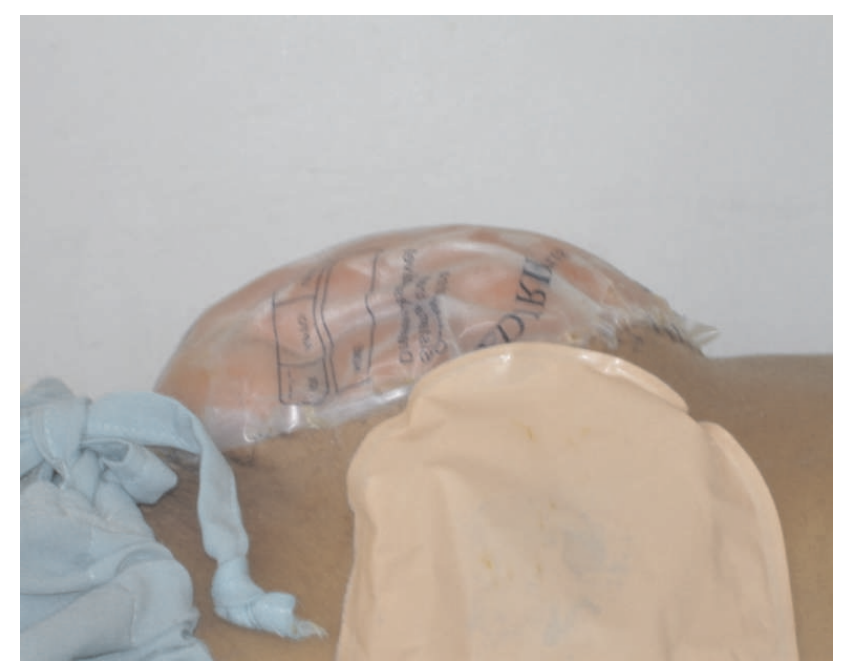

Figura 4 - Fotografia demonstrando paciente em $8^{\circ}$ dia de peritoneostomia com Bolsa de Bogotá, apresentando também ileostomia (visão de perfil).

cientes $^{(3)}$. Em nossa casuística houve fistulização em um $(8,3 \%)$ paciente, provavelmente, conforme relato da literatura, em função da isquemia intestinal. Observamos ainda, que esse risco de fistulização ocorre ainda em função de termos necessidade de dissecar alças e liberar aderências nas sucessivas laparotomias.

Como complicação adicional da técnica, há também o risco aumentado de evisceração: Schachtrupp em $2002{ }^{(3)}$ relata evisceração de $10 \%$ dos pacientes. Nós nos deparamos com a ocorrência de evisceração em dois $(16,7 \%)$ pacientes, um pacien-

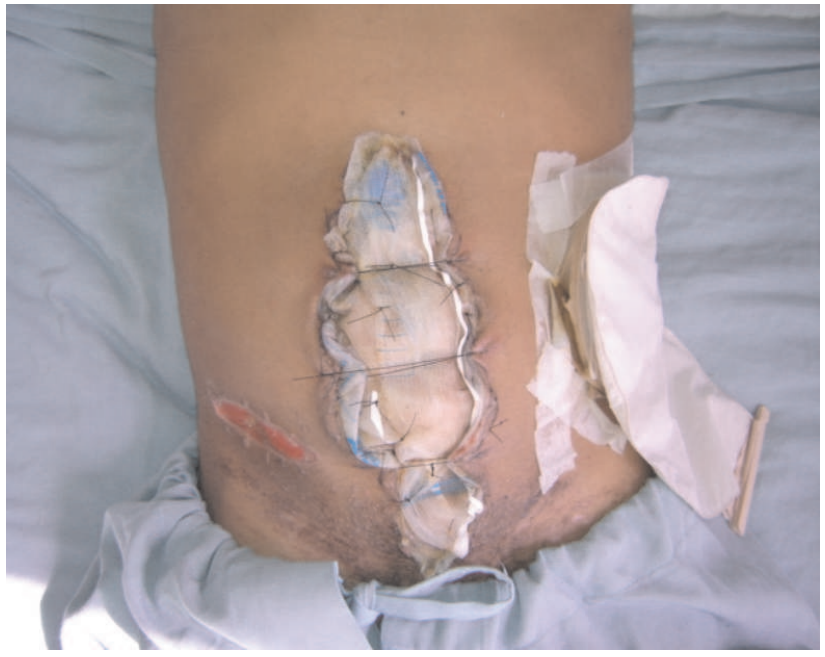

Figura 5 - Fotografia demonstrando o mesmo paciente no $17^{\circ}$ dia de peritoneostomia. Foi realizada uma aproximação parcial dos bordos e utilizada tela de polipropileno como reforço de contenção.

te em uso apenas de bolsa de Bogotá e outro com tela de polipropileno associada.

A literatura relata taxas de mortalidades em trials recentes que vão desde 19\% (Howdieshell em $2004^{(4)}$ ) a 44,7\% ( Tremblay em $2001^{(18)}$ ), Losanoff em $1997^{(10)}$ relatou 20,7\% de mortalidade. Em nossa experiência 4(33,3\%) pacientes vieram a óbito tanto por complicações da peritoneostomia, como também por complicações pulmonares, renais, abdominais ou mesmo falência de múltiplos órgãos, haja vista a utilização do método em pacientes graves.

Outro momento importante a ser decidido quando da utilização da peritoneostomia é o fechamento da parede abdominal. Alguns pontos devem ser levados em consideração, quer sejam: resolução da sepse intraabdominal que ensejou o procedimento, ou a condição clínica do paciente para submeter-se ao tratamento cirúrgico "definitivo", pesados esses fatores deve ser realizado o fechamento tão logo quanto possível devido ao risco de atrofia da parede abdominal ${ }^{(17,18)}$. O fechamento da parede abdominal em nosso estudo, como já citado, foi realizado de 1 a 36 dias com uma média de 10,9 dias (fig.6-7).

Constatamos ser a peritoneostomia um método bastante útil no tratamento da sepse abdominal e a partir dos dados dessa pesquisa estabelecemos um protocolo de acompanhamento prospectivo dos pacientes que venha a utilizar o método, objetivando, na medida do possível, reduzir a morbidade e mortalidade inerentes ao procedimento e a patologia que ensejou o tratamento. 


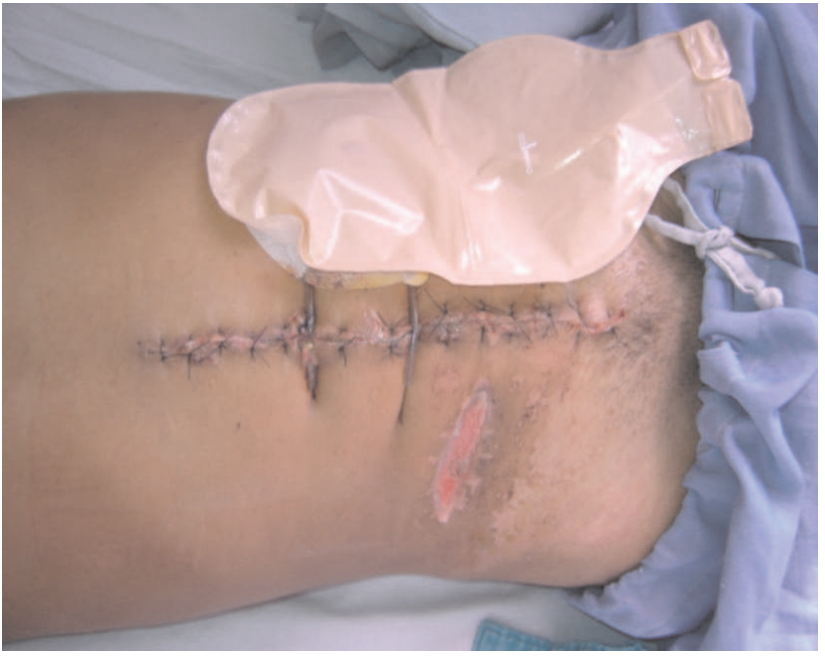

Figura 6- Fotografia demonstrando o fechamento da parede abdominal no $30^{\circ}$ dia de peritoneostomia (visão superior).

Levantaremos ainda a incidência de complicações tardias da peritoneostomia, como, por exemplo, a ocorrência de hérnia ventral incisional.

\section{CONCLUSÃO}

A peritoneostomia é uma arma importante no controle da infecção intra-abdominal, po-

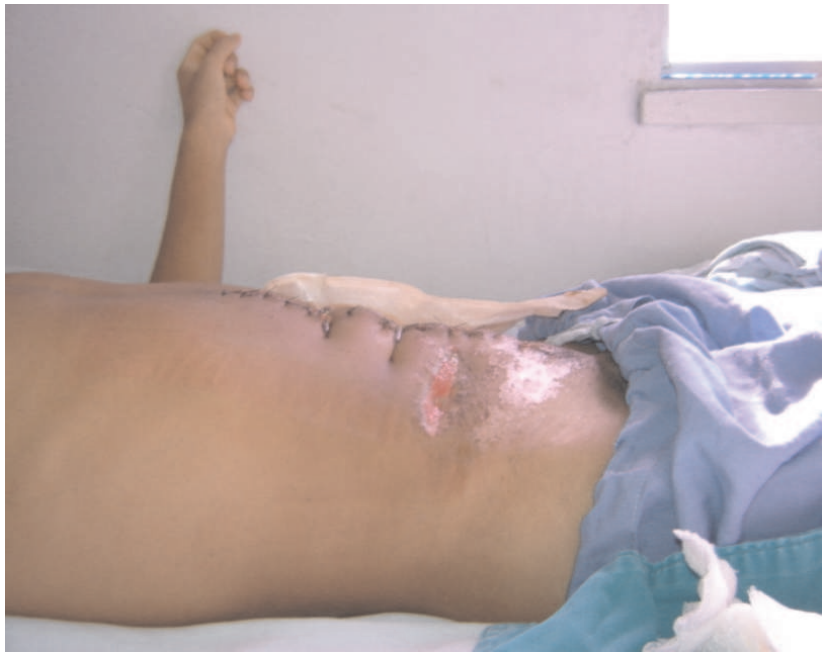

Figura 7 - Fotografia demonstrando o fechamento da parede abdominal no $30^{\circ}$ dia de peritoneostomia (visão perfil).

dendo dar bons resultados na luta contra a síndrome da resposta inflamatória sistêmica nesses pacientes. É um método com risco de complicações, como fístula e eventração, e ainda com mortalidade elevada não apenas imputada à utilização da peritoneostomia, mas também a complicações locais ou gerais devidas à gravidade dos pacientes.

ABSTRACT: Among the therapeutics approach form of abdominal sepsis, the laparostomy has a decisive role allowing cavity explorations and lavages in an easier way. We study patients with abdominal sepsis diagnoses admitted to our surgical service of Coloproctology form Sergipe's Federal University Hospital who underwent a Bogotá Bag laparostomy associated or not with polypropylene mesh from January 2004 to January 2006. These patients were assessed as: first and second diagnosis; secondary peritonitis type; antibiotic-therapy; lavages setup; laparostomy's time; complications and the end of the treatment. We study $10(83.3 \%)$ men and $2(16.7 \%)$ women, with average age of 39,3 (15-57). First diagnosis: inflammatory abdomen acute $6(50 \%)$, block acute abdomen $2(16.7 \%)$, perforative acute abdomen $2(16.7 \%)$, fistula $1(8.3 \%)$ and intracavity abscess $1(8.3 \%)$. Secondary diagnosis: colon perforation in $4(33.3 \%)$, intracavity abscess $3(25 \%)$, anastomoses dehiscence $3(25 \%)$, $1(8.3 \%)$ with sigmoid perforative cancer and $1(8.3 \%)$ with colon necrosis. Fecal peritonitis was found in 10 patients $(83.3 \%)$ and purulent in $2(16.7 \%)$. The antibiotic-therapy was made during nineteen days. Lavages on demand $6(50 \%)$, planned with $4(33.3 \%)$ and mixed setup in 2(16.7\%). The average time of the laparostomy was 10,9 days (1-36). Complications: evisceration in 2 cases (16.7\%) and fistulization in only one $(\mathbf{8 . 3 \%})$. Four patients died.

Key words: Laparostomy, Temporary abdominal closure, Bogotá Bag, Peritonitis, abdominal sepsis.

\section{REFERÊNCIAS}

1. Van Goor H. Interventional management of abdominal sepsis: when and how. Langenbeck's Arch Surg 2002; 387:p 191-2

2. Bailey C M H, Thompson-Fawcett M W, Kettlewell M G W, Chir M, Garrard C, Phil D, Mortensen N J M. Laparostomy for severe intra-abdominal infection complicating colorectal disease. Dis Colon Rectum 2000;43: p 25-30.

3. Schachtrupp A, Fackeldey V, Klinge U, Hoer J, Tittel A, Toens C, Schumpelick V. Temporary closure of the abdominal wall (laparostomy). Hernia 2002; 6: p 155162 . 
4. Howdieshell T R, Proctor C D, Sternberg E, Cue' J I, Mondy J S, Hawkins M L. Temporary abdominal closure followed by definitive abdominal wall reconstruction of the open abdomen. Am J of Surg 2004; 188: p 301-306.

5. Schein M. Planned reoperations and open management in critical intra-abdominal infections: Prospective experience in 52 cases. W J of Surg 1991; 15(4): p 537 - 545

6. Anderson E D, Mandelbaum D M, Ellison E C, Carey L C, Cooperman M. Open packing of the peritoneal cavity in generalized bacterial peritonitis. Am J of Surg 1983; 145(1): p 131-135

7. Losanoff J E, Richman B W, Jones J W. Temporary abdominal coverage and reclosure of the open abdomen: frequently asked questions. J Am Coll Surg 2002;195:p 105-15.

8. Nagy K K, Fildes J J, Mahr C, Roberts R R, Krosner S M, Joseph $\mathrm{K}$ T et al. Experience with three prosthetic materials in temporary abdominal wall closure. Am Surg. 1996 May;62(5):p 331-5.

9. Mayberry J C, Mullins R J, Crass R A, Trunkey D D. Prevention of Abdominal Compartment Syndrome by Absorbable Mesh Prosthesis Closure. Arch Surg.1997;132:p 957-962

10. Losanoff J E, Kjossev K T. Mesh-foil laparostomy. J Am Coll Surg. 1997;185: p 96-99

11. Moore E E, Burch J M, Franciose R J, Offner P J, Biffl W L. Staged Physiologic Restoration and Damage Control Surgery. World J. Surg. 1998;22: p1184-1191

12. Alfici A, Ashkenazi I, Kessel B, Zut N, Sternberg A. Temporary Bowel Diversion Using the Bogota' Bag (Hadera Stoma): Technical Details. J Am Coll Surg 2004;199(2)
13. Navsaria P H, Bunting M, Omoshoro-Jones J, Nicol A J, Kahn D. Temporary closure of open abdominal wounds. British Journal of Surgery 2003; 90: 718-722

14. Letoublon C, Cardin N, Arvieux C. La laparostomie par pansement pariétal aspiratif ou «Vacuum Pack» Laparostomy with vacuum pack technique. Annales de chirurgie 2005; 130: p 587-589

15. Hedderich G S, Wexler M J, McLean A P, Meakins J L. The septic abdomen: open management with Marlex mesh with a zipper. Surgery 1986; 99(4): p 399-408

16. Bose S M, Kalra M, Sandhu N P. Open management of septic abdomen by Marlex mesh zipper. Aust N Z J Surg 1991; 61(5): p 385-8

17. Schachtrupp A, Hoer J, Tons C, Klinge U, Reckord U, Schumpelick V. Intra-abdominal pressure: a reliable criterion for laparostomy closure?. Hernia. 2002; 6(3): p 102-7

18. Tremblay L N, Feliciano D V, Cava J S R A, Tchorz K M, Ingram W L, Salomone J P et al. Skin only or silo closure in the critically ill patient with an open abdomen. Am J of Surg 2001; 182: p 670-675.

\section{Endereço para correspondência:}

\section{JUVENAL DA ROCHA TORRES NETO}

Rua Ananias Azevedo, 100, Ed. Piazza Fiorentina, apto. 902

Treze de Julho, Aracaju - SE - Brasil

Fax: (79) $3214-4830$

E-mail: jtorres@infonet.com.br 